\title{
Effects of vacuum structure on neutron stars
}

\author{
P. K. Panda, ${ }^{1, *}$ R. Sahu, ${ }^{2}$ and S. Mishra ${ }^{2}$ \\ ${ }^{1}$ Instituto de Física Teorica, Universidade Estadual Paulista, Rua Pamplona 145, 01405-900 Sao Paulo - SP, Brazil \\ ${ }^{2}$ Department of Physics, Berhampur University, Berhampur-760007, Orissa, India
}

(Received 11 July 2000; published 10 October 2000)

\begin{abstract}
We study the equation of state for neutron matter using the Walecka model including quantum corrections for baryons and sigma mesons through a realignment of the vacuum. We next use this equation of state to calculate the radius, mass, and other properties of rotating neutron stars.
\end{abstract}

PACS number(s): 26.60.+c, 21.65.+f, 21.30.-x, 97.60.Jd

\section{INTRODUCTION}

Neutron stars formed after a supernova explosion are the smallest, densest stars known. At the time of their birth, they are composed of supernova matter at high temperature. However, they rapidly cool down by the neutrino diffusion process. Hence most of the observed neutron stars are essentially cold objects. The equation of state is the essential ingredient for studying such neutron stars. Since the central density of a neutron star is so large, one should determine the equation of state using a relativistic model.

In recent years there has been considerable progress in the study of nuclear matter both at zero temperature [1] as well as at high temperature. In all these considerations relativistic mean-field theory using hadronic degrees of freedom has been applied for the study of the ground-state properties of nuclear matter. For example in the Walecka model [1], the nucleons interact through the exchange of scalar $(\sigma)$ and vector $(\omega)$ mesons. In this model it has been possible to describe the saturation density and binding energy of nuclear matter by adjusting the scalar and vector couplings. However, in this treatment the effect of the Dirac sea has been neglected. Effects associated with the Dirac sea of nucleons have been proposed [2] as important to several nuclear physics problems. The simplest interpretation of these effects is in terms of virtual $N \bar{N}$ pairs. It has been argued [2] that the composite nature of the nucleon probably supress the contributation of $N \bar{N}$ pairs compared to what is expected in naive Dirac hole theory. It has also shown [2] that one-nucleonloop contributions in quantum hydrodynamics do appear to be suppressed. Glendenning [3] has performed a vacuum renormalization of relativistic field theory to study the properties of neutron stars. There are also attempts to include the contribution of the filled negative sea of nucleons in nuclear matter at the one- and two-loop levels $[4,5]$. However, it is found that the binding energy up to two loops [5] is rather large as compared to the one-loop results. This might be because the couplings involved here are too large (of the order of 10) and the theory is not assymptotically free. Hence nonperturbative techniques need to consider the many-body problem. In a recent study [6], vacuum polarization correction is included in nuclear matter in a nonperturbative ap-

*Electronic address: panda@ift.unesp.br proach. In this study, one uses a squeezed coherent type of construction for the ground state which amounts to an explicit vacuum realignment. It was earlier seen that the method correctly yields the results of the Gross-Neveu [7] model as obtained by summing an infinite series of one-loop diagrams. It has also studied that it reproduces the gap equation in an effective QCD Hamiltonian [8] as obtained through solution of the Schwinger-Dyson equation for the effective quark propagator. We here apply the same techniques to study the quantum vacuum. The input here is equal-time quantum algebra for the field operators with a variational ansatz for the vacuum structure. Without using any perturbative expansion or Feynman diagrams, it has been possible to include quantum corrections through a realignment of the ground state with baryon as well as $\sigma$-meson condensates. The only baryon condensates yield the same result as obtained in the relativistic Hartree approximation (RHA) and give a softer equation of state as compared to the no-sea approximation. The equation of state obtained in this approach is expected to be quite reliable. Hence it would be quite interesting to extend this model to neutron matter by including the $\rho$ meson and to use the equation of state so obtained to study the properties of rotating neutron star.

We organize the paper as follows. In Sec. II, we give a brief description of the model. The results are discussed in Sec. III. The conclusions drawn from the present study are presented in Sec. IV.

\section{THEORY}

\section{A. Vacuum with condensates}

The details of the theory have already been discussed in Ref. [6]. Only a few important steps are given here.

We start with the Lagrangian density for the linear Walecka model [1] given as

$$
\begin{aligned}
\mathcal{L}= & \bar{\psi}\left(i \gamma^{\mu} \partial_{\mu}-M-g_{\sigma} \sigma-g_{\omega} \gamma^{\mu} \omega_{\mu}-\frac{1}{2} g_{\rho} \gamma_{\mu} \boldsymbol{\tau} \cdot \mathbf{R}^{\mu}\right) \psi \\
& +\frac{1}{2} \partial^{\mu} \sigma \partial_{\mu} \sigma-\frac{1}{2} m_{\sigma}^{2} \sigma^{2}+\frac{1}{2} m_{\omega}^{2} \omega^{\mu} \omega_{\mu}-\frac{1}{4} \omega^{\mu \nu} \omega_{\mu \nu} \\
& +\frac{1}{2} m_{\rho}^{2} \mathbf{R}^{\mu} \mathbf{R}_{\mu}-\frac{1}{4} \mathbf{R}^{\mu \nu} \mathbf{R}_{\mu \nu},
\end{aligned}
$$




$$
\begin{aligned}
& \omega_{\mu \nu}=\partial_{\mu} \omega_{\nu}-\partial_{\nu} \omega_{\mu}, \\
& \mathbf{R}_{\mu \nu}=\partial_{\mu} \mathbf{R}_{\nu}-\partial_{\nu} \mathbf{R}_{\mu} .
\end{aligned}
$$

In the above, $\psi, \sigma, \omega_{\mu}$, and $\mathbf{R}_{\mu}$ are the fields for the baryons, $\sigma, \omega$, and $\rho$ mesons with masses $M, m_{\sigma}, m_{\omega}$, and $m_{\rho}$, respectively. We use the mean-field approximation for the meson fields and retain the quantum nature of the fermion fields. This amounts to taking meson fields as constant classical fields with translational invariance for neutron matter.

The Hamiltonian density can then be written as

$$
\mathcal{H}=\mathcal{H}_{N}+\mathcal{H}_{\sigma}+\mathcal{H}_{\omega}+\mathcal{H}_{\rho}
$$

with

$$
\begin{gathered}
\mathcal{H}_{N}=\psi^{\dagger}(-i \boldsymbol{\alpha} \cdot \nabla+\beta M) \psi+g_{\sigma} \sigma \bar{\psi} \psi, \\
\mathcal{H}_{\sigma}=\frac{1}{2} m_{\sigma}^{2} \sigma^{2}, \\
\mathcal{H}_{\omega}=g_{\omega} \omega_{0} \psi^{\dagger} \psi-\frac{1}{2} m_{\omega}^{2} \omega_{0}^{2}, \\
\mathcal{H}_{\rho}=\frac{1}{2} m_{\rho}^{2} R_{03}^{2} .
\end{gathered}
$$

The equal-time quantization condition for the baryons is given as

$$
\left[\psi_{\alpha_{1}}(\mathbf{x}, t), \psi_{\alpha_{2}}^{\dagger}(\mathbf{y}, t)\right]_{+}=\delta_{\alpha_{1} \alpha_{2}} \delta(\mathbf{x}-\mathbf{y})
$$

where $\alpha_{1}$ and $\alpha_{2}$ refer to the spin indices. We may now write down the field expansion for the baryon field $\psi$ at time $t$ $=0$ as given by [9]

$$
\psi(\mathbf{x})=\frac{1}{(2 \pi)^{3 / 2}} \int\left[U_{r}(\mathbf{k}) c_{I r}(\mathbf{k})+V_{s}(-\mathbf{k}) \tilde{c}_{I s}(-\mathbf{k})\right] e^{i \mathbf{k} \cdot \mathbf{x}} d \mathbf{k},
$$

with $c_{I r}$ and $\tilde{c}_{I s}$ as the baryon annihilation and antibaryon creation operators with spins $r$ and $s$, respectively. In the above, $U_{r}$ and $V_{s}$ are given by

$$
\begin{gathered}
U_{r}(\mathbf{k})=\left(\begin{array}{c}
\cos \frac{\chi(\mathbf{k})}{2} \\
\boldsymbol{\sigma} \cdot \hat{\mathbf{k}} \sin \frac{\chi(\mathbf{k})}{2}
\end{array}\right) u_{I r}, \\
V_{s}(-\mathbf{k})=\left(\begin{array}{c}
-\boldsymbol{\sigma} \cdot \hat{\mathbf{k}} \sin \frac{\chi(\mathbf{k})}{2} \\
\cos \frac{\chi(\mathbf{k})}{2}
\end{array}\right) v_{I s} .
\end{gathered}
$$

In the above $\boldsymbol{\sigma}$ is the Pauli spin matices and $\hat{\mathbf{k}}=\mathbf{k} /|\mathbf{k}|$. For free massive fields $\cos \chi(\mathbf{k})=M / \epsilon(\mathbf{k})$ and $\sin \chi(\mathbf{k})$ $=|\mathbf{k}| / \epsilon(\mathbf{k})$, with $\epsilon(\mathbf{k})=\sqrt{\mathbf{k}^{2}+M^{2}}$. The perturbative vacuum, say $|\mathrm{vac}\rangle$, is defined through $c_{I r}(\mathbf{k})|\mathrm{vac}\rangle=0$ and $\tilde{c}_{I r}^{\dagger}(\mathbf{k})|\mathrm{vac}\rangle=0$. To include the vacuum-polarization effects, we shall now consider a trial state with baryon-antibaryon condensates. We thus explicitly take the ansatz for the above state as

$$
\begin{aligned}
\left|\operatorname{vac}^{\prime}\right\rangle & =\exp \left[\int d \mathbf{k} f(\mathbf{k}) c_{I r}^{\dagger}(\mathbf{k}) a_{r s} \tilde{c}_{I s}(-\mathbf{k})-\text { H.c. }\right]|\mathrm{vac}\rangle \\
& \equiv U_{F}|\mathrm{vac}\rangle .
\end{aligned}
$$

Here $a_{r s}=u_{I r}^{\dagger}(\boldsymbol{\sigma} \cdot \hat{\mathbf{k}}) v_{I s}$ and $f(\mathbf{k})$ is a trial function associated with baryon-antibaryon condensates. We note that with the above transformation the operators corresponding to $\left|\mathrm{vac}^{\prime}\right\rangle$ are related to the operators corresponding to $|\mathrm{vac}\rangle$ through the Bogoliubov transformation. We then use the method of thermofield dynamics [10] developed by Umezawa and coworkers to construct the ground state for nuclear matter. We generalize the state with baryon-antibaryon condensates as given by Eq. (8) to finite temperature and density as [6]

$$
|F(\beta)\rangle=U(\beta)\left|\operatorname{vac}^{\prime}\right\rangle \equiv U(\beta) U_{F}|\operatorname{vac}\rangle .
$$

The temperature-dependent unitary operator $U(\beta)$ is given as [10]

$$
U(\beta)=\exp \left[A^{\dagger}(\beta)-A(\beta)\right]
$$

with

$$
\begin{aligned}
A^{\dagger}(\beta)= & \int d \mathbf{k}\left[\theta_{-}(\mathbf{k}, \beta) d_{I r}^{\dagger}(\mathbf{k}) \underline{d}_{-}^{\dagger}(-\mathbf{k})\right. \\
& \left.+\theta_{+}(\mathbf{k}, \beta) \widetilde{d}_{I r}(\mathbf{k}) \widetilde{d}_{I r}(-\mathbf{k})\right] .
\end{aligned}
$$

The underlined operators are operators corresponding to the doubling of the Hilbert space that arises in the thermofield dynamics method. We shall determine the condensate function $f(\mathbf{k})$ and the functions $\theta_{-}(\mathbf{k}, \beta)$ and $\theta_{+}(\mathbf{k}, \beta)$ of the thermal vacuum through minimization of the thermodynamic potential. The energy density is given as

$$
\epsilon \equiv\langle\mathcal{H}\rangle_{\beta}
$$

and the baryon density

$$
\rho_{B}=\frac{g}{(2 \pi)^{3}} \int d \mathbf{k}\left(\cos ^{2} \theta_{+}+\sin ^{2} \theta_{-}\right)
$$

In the above, $g$ is the spin isospin degeneracy factor and is equal to 4 for nuclear matter, 2 for neutron matter.

Extremizing the thermodynamic potential with respect to the condensate function $f(\mathbf{k})$ and the functions $\theta_{\mp}$ yields

$$
\tan 2 f(\mathbf{k})=\frac{g_{\sigma} \sigma_{0}|\mathbf{k}|}{\epsilon(k)^{2}+M g_{\sigma} \sigma_{0}}
$$

and 


$$
\sin ^{2} \theta_{\mp}=\frac{1}{\exp \left\{\beta\left[\epsilon^{*}(\mathbf{k}) \mp \mu^{*}\right]\right\}+1},
$$

with $\epsilon^{*}(\mathbf{k})=\left(\mathbf{k}^{2}+M^{* 2}\right)^{1 / 2}$ and $\mu^{*}=\mu-g_{\omega} \omega_{0}-\frac{1}{2} g_{\rho} R_{03}$ as the effective energy density and effective chemical potential, where the effective baryon mass is $M^{*}=M+g_{\sigma} \sigma_{0}$.

We now proceed to study the equation of state for neutron matter at zero temperature. The energy density after subtracting out the pure vacuum contribution then becomes

$$
\epsilon_{0} \equiv \epsilon\left(\theta_{-}, f\right)-\epsilon\left(\theta_{-}=0, f=0\right)=\epsilon_{M F T}+\Delta \epsilon,
$$

with

$$
\begin{aligned}
\epsilon_{M F T}= & \frac{g}{(2 \pi)^{3}} \int_{|\mathbf{k}|<k_{F}} d \mathbf{k}\left(k^{2}+M^{* 2}\right)^{1 / 2}+\frac{1}{2} m_{\sigma}^{2} \sigma_{0}^{2}+\frac{1}{2} m_{\omega}^{2} \omega_{0}^{2} \\
& +\frac{1}{2} m_{\rho}^{2} R_{03}^{2}
\end{aligned}
$$

and

$$
\begin{aligned}
\Delta \epsilon= & -\frac{g}{(2 \pi)^{3}} \int d \mathbf{k}\left[\left(k^{2}+M^{* 2}\right)^{1 / 2}-\left(k^{2}+M^{2}\right)^{1 / 2}\right. \\
& \left.-\frac{g_{\sigma} \sigma_{0} M}{\left(k^{2}+M^{2}\right)^{1 / 2}}\right] .
\end{aligned}
$$

The above expression for the energy density is divergent. After renormalization [11] by adding the counterterms, we have the expression for the finite renormalized energy density:

$$
\epsilon_{r e n}=\epsilon_{M F T}+\Delta \epsilon_{r e n},
$$

where

$$
\begin{aligned}
\Delta \epsilon_{r e n}= & -\frac{g}{16 \pi^{2}}\left[M^{* 4} \ln \left(\frac{M^{*}}{M}\right)+M^{3}\left(M-M^{*}\right)\right. \\
& -\frac{7}{2} M^{2}\left(M-M^{*}\right)^{2}+\frac{13}{3} M\left(M-M^{*}\right)^{3} \\
& \left.-\frac{25}{12} M\left(M-M^{*}\right)^{4}\right] .
\end{aligned}
$$

The thermodynamic potential is a function of $\sigma_{0}, \omega_{0}$, and $R_{03}$. This when minimized with respect to $\sigma_{0}$ gives the selfconsistency condition for the effective baryon mass,

$$
M^{*}=M-\frac{g_{\sigma}^{2}}{m_{\sigma}^{2}} \frac{g}{(2 \pi)^{3}} \int^{k_{F}} d \mathbf{k} \frac{M^{*}}{\epsilon(k)^{*}}+\Delta M^{*},
$$

where

$$
\begin{aligned}
\Delta M^{*}= & \frac{g_{\sigma}^{2}}{m_{\sigma}^{2}} \frac{g}{(2 \pi)^{3}}\left[M^{* 3} \ln \left(\frac{M^{*}}{M}\right)+M^{2}\left(M-M^{*}\right)\right. \\
& \left.-\frac{5}{2} M^{2}\left(M-M^{*}\right)^{2}+\frac{11}{6} M\left(M-M^{*}\right)^{3}\right] .
\end{aligned}
$$

We next consider the quantum corrections due to the scalar mesons. They arise from a vacuum realignment with sigma condensates, meaning thereby that the $\sigma$ field is not a classical one, but a quantum field. As will be seen later, a quartic term in the sigma field would favor such condensates. Self-interactions of scalar fields with cubic and quartic terms have been considered earlier [12] in the no-sea approximation [13] as well as including the quantum corrections arising from the sigma fields $[1,3]$. They may be regarded as mediating three- and four-body interactions between the baryons. The best fits to incompressibility in nuclear matter, singleparticle spectra, and properties of deformed nuclei are achieved with a negative value for the quartic coupling in the sigma field. However, with such a negative coupling the energy spectrum of the theory becomes unbounded from below [14] for large $\sigma$ and hence it is impossible to study excited spectra or to include vacuum polarization effects.

Including a quartic scalar self-interaction, Eq. (4b) is modified to

$$
\mathcal{H}_{\sigma}=\frac{1}{2} \partial_{\mu} \sigma \partial^{\mu} \sigma+\frac{1}{2} m_{\sigma}^{2} \sigma^{2}+\lambda \sigma^{4},
$$

with $m_{\sigma}$ and $\lambda$ being the bare mass and coupling constant, respectively. The $\sigma$ field satisfies the quantum algebra

$$
[\sigma(\mathbf{x}), \dot{\sigma}(\mathbf{y})]=i \delta(\mathbf{x}-\mathbf{y}) .
$$

We may expand the field operators in terms of creation and annihilation operators at time $t=0$ as

$$
\begin{gathered}
\sigma(\mathbf{x}, 0)=\frac{1}{(2 \pi)^{3 / 2}} \int \frac{d \mathbf{k}}{\sqrt{2 \omega(\mathbf{k})}}\left[a(\mathbf{k})+a^{\dagger}(-\mathbf{k})\right] e^{i \mathbf{k} \cdot \mathbf{x}}, \\
\dot{\sigma}(\mathbf{x}, 0)=\frac{i}{(2 \pi)^{3 / 2}} \int d \mathbf{k} \sqrt{\frac{\omega(\mathbf{k})}{2}}\left[-a(\mathbf{k})+a^{\dagger}(-\mathbf{k})\right] e^{i \mathbf{k} \cdot \mathbf{x}} .
\end{gathered}
$$

In the above, $\omega(\mathbf{k})$ is an arbitrary function which for free fields is given by $\omega(\mathbf{k})=\sqrt{\mathbf{k}^{2}+m_{\sigma}^{2}}$ and the perturbative vacuum is defined corresponding to this basis through $a|\mathrm{vac}\rangle=0$. As seen earlier a realignment of the ground state from $|v a c\rangle$ to $\left|v \mathrm{vac}^{\prime}\right\rangle$ with baryon condensates amounts to including quantum effects. We shall adopt a similar procedure now to calculate the quantum corrections arising from the $\sigma$ field. We thus modify the ansatz for the trial ground state as given by Eq. (8) to include $\sigma$ condensates as [15]

$$
|\Omega\rangle=U_{\sigma} U_{F}|\mathrm{vac}\rangle,
$$

with 


$$
U_{\sigma}=U_{I I} U_{I},
$$

where $U_{i}=\exp \left(B_{i}^{\dagger}-B_{i}\right), \quad(i=I, I I)$. Explicitly the $B_{i}$ are given as

$$
B_{I}^{\dagger}=\int d \mathbf{k} \sqrt{\frac{\omega(\mathbf{k})}{2}} f_{\sigma}(\mathbf{k}) a^{\dagger}(\mathbf{k})
$$

and

$$
B_{I I}^{\dagger}=\frac{1}{2} \int d \mathbf{k} g(\mathbf{k}) a^{\prime \dagger}(\mathbf{k}) a^{\prime \dagger}(-\mathbf{k}) .
$$

In the above, $a^{\prime}(\mathbf{k})=U_{I} a(\mathbf{k}) U_{I}^{-1}=a(\mathbf{k})-[\sqrt{\omega(\mathbf{k}) / 2}] f_{\sigma}(\mathbf{k})$ corresponds to a shifted field operator associated with the coherent state [15] and satisfies the same quantum algebra as $a(\mathbf{k})$. Thus in this construct for the ground state we have two functions $f_{\sigma}(\mathbf{k})$ and $g(\mathbf{k})$ which will be determined through minimization of energy density. Further, since $|\Omega\rangle$ contains an arbitrary number of $a^{\prime \dagger}$ quanta, $a^{\prime}|\Omega\rangle \neq 0$. However, we can define the basis $b(\mathbf{k}), b^{\dagger}(\mathbf{k})$ corresponding to $|\Omega\rangle$ through the Bogoliubov transformation as

$$
b(\mathbf{k})=U_{I I} a^{\prime}(\mathbf{k}) U_{I I}^{-1}=(\cosh g) a^{\prime}(\mathbf{k})-(\sinh g) a^{\prime \dagger}(-\mathbf{k}) .
$$

It is easy to check that $b(\mathbf{k})|\Omega\rangle=0$. Further, to preserve translational invariance $f_{\sigma}(\mathbf{k})$ has to be proportional to $\delta(\mathbf{k})$ and we take $f_{\sigma}(\mathbf{k})=\sigma_{0}(2 \pi)^{3 / 2} \delta(\mathbf{k})$. Here $\sigma_{0}$ will correspond to a classical field of the conventional approach [15]. We next calculate the expectation value of the Hamiltonian density. We use the renormalization prescription of Ref. [16] and thus obtain the gap equation for $M_{\sigma}^{2}$ in terms of the renormalized parameters as

$$
M_{\sigma}^{2}=m_{R}^{2}+12 \lambda_{R} \sigma_{0}^{2}+12 \lambda_{R} I_{f}\left(M_{\sigma}\right),
$$

where

$$
I_{f}\left(M_{\sigma}\right)=\frac{M_{\sigma}^{2}}{16 \pi^{2}} \ln \left(\frac{M_{\sigma}^{2}}{\mu^{2}}\right) .
$$

Then using the above equations we obtain the energy density for the $\sigma$ in terms of $\sigma_{0}$ as

$$
\begin{aligned}
\epsilon_{\sigma}= & 3 \lambda_{R}\left(\sigma_{0}^{2}+\frac{m_{R}^{2}}{12 \lambda_{R}}\right)^{2}+\frac{M_{\sigma}^{4}}{64 \pi^{2}}\left[\ln \left(\frac{M_{\sigma}^{2}}{\mu^{2}}\right)-\frac{1}{2}\right] \\
& -3 \lambda_{R} I_{f}^{2}-2 \lambda \sigma_{0}^{4} .
\end{aligned}
$$

The above expression is given in terms of the renormalized $\sigma$ mass $m_{R}$ and the renormalized coupling $\lambda_{R}$ except for the last term which is still in terms of the bare coupling constant $\lambda$ and did not get renormalized because of the structure of the gap equation [17]. However, from the renormalization procedure it is easy to see that when $\lambda_{R}$ is kept fixed, the bare coupling $\lambda \rightarrow 0_{-}$. Therefore the last term in Eq. (32) will be neglected in the numerical calculations.

After subtracting the vacuum contribution, we get

$$
\begin{aligned}
\Delta \epsilon_{\sigma}= & \epsilon_{\sigma}-\epsilon_{\sigma}\left(\sigma_{0}=0\right) \\
= & \frac{1}{2} m_{R}^{2} \sigma_{0}^{2}+3 \lambda_{R} \sigma_{0}^{4}+\frac{M_{\sigma}^{4}}{64 \pi^{2}}\left[\ln \left(\frac{M_{\sigma}^{2}}{\mu^{2}}\right)-\frac{1}{2}\right] \\
& -3 \lambda_{R} I_{f}^{2}-\frac{M_{\sigma, 0}^{4}}{64 \pi^{2}}\left[\ln \left(\frac{M_{\sigma, 0}^{2}}{\mu^{2}}\right)-\frac{1}{2}\right]+3 \lambda_{R} I_{f 0}^{2},
\end{aligned}
$$

where $M_{\sigma, 0}$ and $I_{f 0}$ are the expressions as given by Eqs. (30) and (31) with $\sigma_{0}=0$.

The energy density and pressure with baryon and sigma condensates are

$$
\epsilon_{\text {ren }}=\epsilon_{0}^{\text {finite }}+\Delta \epsilon_{\text {ren }}
$$

and

$$
\begin{aligned}
P= & \frac{g}{3(2 \pi)^{3}} \int_{|\mathbf{k}|<k_{F}} d \mathbf{k} \frac{k^{2}}{\left(k^{2}+M^{* 2}\right)^{1 / 2}}+\frac{1}{2} m_{\omega}^{2} \omega_{0}^{2}-\Delta \epsilon_{\sigma} \\
& -\Delta \epsilon_{r e n}+\frac{1}{2} m_{\rho}^{2} R_{03}^{2},
\end{aligned}
$$

where

$$
\begin{aligned}
\epsilon_{0}^{\text {finite }}= & \frac{g}{(2 \pi)^{3}} \int_{|\mathbf{k}|<k_{F}} d \mathbf{k}\left(k^{2}+M^{* 2}\right)^{1 / 2}+\frac{1}{2} m_{\omega}^{2} \omega_{0}^{2}+\Delta \epsilon_{\sigma} \\
& +\frac{1}{2} m_{\rho}^{2} R_{03}^{2},
\end{aligned}
$$

with $\Delta \epsilon_{r e n}$ given by Eq. (20).

The energy density from the $\sigma$ field as given by Eq. (33) is still in terms of the renormalization scale $\mu$ which is arbitrary. We choose this to be equal to the renormalized sigma mass $m_{R}$ in doing the numerical calculations. This is because changing $\mu_{B}$ would mean changing the quartic coupling $\lambda_{R}$, and $\lambda_{R}$ here enters as a parameter to be chosen to give the incompressibility for nuclear matter in the correct range. The parameters $g_{\sigma}, g_{\omega}$, and $g_{\rho}$ are fitted so as to describe the ground-state properties of nuclear matter correctly. For a given baryon density $\rho_{B}$, the energy density and the pressure for neutron matter are calculated at zero temperature. We then use this equation of state to calculate the neutron star properties.

\section{B. Neutron star}

The stars are assumed to be stationarily rotating and hence have axially, equatorially symmetric structures. The details of the model are given by Kamatsu et al. [18], Cook et al. [19], and Stergioulas and Friedman [20]. The metric can be written in spherical coordinates $(t, r, \theta, \phi)$ :

$$
\begin{aligned}
d s^{2}= & -e^{2 v} d t^{2}+e^{2 \alpha}\left(d r^{2}+r^{2} d \theta^{2}\right) \\
& +e^{2 \beta} r^{2} \sin ^{2} \theta(d \phi-\omega d t)^{2},
\end{aligned}
$$


where $\alpha, \beta, \nu$, and $\omega$ are the potentials which depend only on $r$ and $\theta$. The geometrized units $c$ and $G$ have been set to unity. The stellar matter is assumed to be a perfect fluid so that the energy momentum tensor $T^{a b}$ is given by

$$
T^{a b}=(\epsilon+P) U^{a} U^{b}+P g^{a b},
$$

where $\epsilon, P, U^{a}$, and $g^{a b}$ are the energy density, pressure, four-velocity, and metric tensor, respectively. It is further assumed that the four-velocity $U^{a}$ is simply a linear combination of the time and angular Killing vectors. The Einstein equations for $\nu, \beta$, and $\omega$ are written as

$$
\begin{gathered}
\Delta\left[\rho e^{\gamma / 2}\right]=S_{\rho}(r, \mu), \\
\left(\Delta+\frac{1}{r} \frac{\partial}{\partial r}-\frac{1}{r^{2}} \mu \frac{\partial}{\partial \mu}\right) \gamma e^{\gamma / 2}=S_{\gamma}(r, \mu), \\
\left(\Delta+\frac{2}{r} \frac{\partial}{\partial r}-\frac{2}{r^{2}} \mu \frac{\partial}{\partial \mu}\right) \omega e^{(\gamma-2 \rho) / 2}=S_{\omega}(r, \mu),
\end{gathered}
$$

where

$$
\begin{aligned}
\Delta=\frac{\partial^{2}}{\partial r^{2}}+\frac{2}{r} \frac{\partial}{\partial r}+\frac{1}{r^{2}} \frac{\partial^{2}}{\partial \theta^{2}} & +\frac{1}{r^{2}} \cot \theta \frac{\partial}{\partial \theta}+\frac{1}{r^{2} \sin ^{2} \theta} \frac{\partial^{2}}{\partial \phi^{2}} \\
\gamma & =\beta+\nu \\
\rho & =\nu-\beta .
\end{aligned}
$$

The detailed expressions for the source terms $S_{\rho}, S_{\gamma}$, and $S_{\omega}$ are given in [18]. The above differential equations can be transformed into an integral representation so as to enable us to handle boundary conditions in a much easier manner. Using a three-dimensional Green's function and introducing cylindrical coordinates $\bar{\omega}=r \sin \theta$ and $z=r \cos \theta$, the integral equations are given as

$$
\begin{aligned}
\rho= & -\sum_{n=0}^{\infty} e^{-\gamma / 2} \int_{0}^{\infty} d r^{\prime} \int_{0}^{1} d \mu^{\prime} r^{\prime 2} f_{2 n}^{2}\left(r, r^{\prime}\right) \\
& \times P_{2 n}(\mu) P_{2 n}\left(\mu^{\prime}\right) S_{\rho}\left(r^{\prime}, \mu^{\prime}\right), \\
r \sin \theta \gamma= & -\frac{2}{\pi} e^{-\gamma / 2} \sum_{n=1}^{\infty} \int_{0}^{\infty} d r^{\prime} \int_{0}^{1} d \mu^{\prime} r^{\prime 2} f_{2 n-1}^{1}\left(r, r^{\prime}\right) \\
& \times \frac{1}{2 n-1} \sin (2 n-1) \theta \sin (2 n-1) \theta^{\prime} S_{\gamma}\left(r^{\prime}, \mu^{\prime}\right),
\end{aligned}
$$

$$
\begin{aligned}
r \sin \theta \omega= & -\sum_{n=1}^{\infty} e^{(2 \rho-\gamma) / 2} \int_{0}^{\infty} d r^{\prime} \int_{0}^{1} d \mu^{\prime} r^{\prime 3} \sin \theta^{\prime} \\
& \times f_{2 n-1}^{2}\left(r, r^{\prime}\right) \frac{1}{2 n(2 n-1)} P_{2 n-1}^{1}(\mu) \\
& \times P_{2 n-1}^{1}\left(\mu^{\prime}\right) S_{\omega}\left(r^{\prime}, \mu^{\prime}\right) .
\end{aligned}
$$

Here $P_{n}$ is the Legendre polynomial, and $P_{n}^{m}$ is the associated Legendre function. The expression for $f_{n}^{1}\left(r, r^{\prime}\right)$ and $f_{n}^{2}\left(r, r^{\prime}\right)$ are given in [18]. The expressions for the potential $\alpha$ is also given in Ref. [18]. Thus the calculation involves solving the four field equations for $\rho, \gamma, \omega$, and $\alpha$.

\section{RESULTS AND DISCUSSION}

As has been discussed in Ref. [6], the numerical calculation at zero temperature is carried out in the following steps: The masses of the nucleons, $\omega$ mesons, and $\rho$ mesons are taken to be 939,783 , and $770 \mathrm{MeV}$, respectively. Then we calculate the binding energy per nucleon for a given renormalized $\sigma$ mass $m_{R}$ and renormalized coupling $\lambda_{R}$ and fit the corresponding scalar and vector couplings $g_{\sigma}$ and $g_{\omega}$ to get the correct saturation properties for symmetric nuclear matter. $g_{\rho}$ is fixed so as to reproduce the asymmetry energy of $32.5 \mathrm{MeV}$ for a given $m_{R}$ and $\lambda_{R}$. We have tabulated the masses and the coupling constants of $\sigma, \omega$, and $\rho$ mesons in Table I. We have also given the compressibility and the effective mass for different $\lambda_{R}$ in Table I. The compressibility and the effective mass lie in the range of $300-400 \mathrm{MeV}$ and $0.75-0.8$, respectively. It is seen that the coupling $g_{\sigma}$ and $g_{\omega}$ decrease with increasing $\lambda_{R}$. However, $g_{\rho}$ increases with $\lambda_{R}$.

For $m_{R}=520 \mathrm{MeV}$, the equation of state for neutron matter is calculated for different $\lambda_{R}$ and plotted in Fig. 1. We have also plotted the equation of state for the Walecka model where the contribution of the Dirac sea has been neglected. It is seen that the equation of state becomes stiff if the Dirac sea is neglected. The equation of state for the relativistic Hartree approximation is also shown. With the increase of $\lambda_{R}$, the equation of state becomes softer. As a result, the compressibility goes on decreasing with $\lambda_{R}$ as shown in Table I.

We next study neutron star properties using the above equation of state. The maximum mass of the neutron star, known as limiting mass, is interesting because this can be measured on binary systems. The most massive measurement is for 4U0900-40 with $M=1.85_{-0.30}^{+0.35} M_{\odot}$ and the most accurate is for PSR1913+16 with $M=1.451 \pm 0.007 M_{\odot}$ [21]. In Fig. 2, we plot the mass of the neutron star as a function of the radius of the neutron star for different $\lambda_{R}$ with $m_{R}=520 \mathrm{MeV}$. The no-sea approximation predicts a much larger radius and maximum mass for the neutron star. However, with the increase of $\lambda_{R}$, both the maximum stable mass and radius of the neutron star decrease.

The equation of state and the structure of the neutron stars are suggested in Ref. [22] using the variational chain summation methods and the new Argonne $v_{18}$ two-nucleon in- 
TABLE I. Mass of the nucleon $M, \omega$ meson $m_{\omega}, \rho$ meson $m_{\rho}$, and $\sigma$ meson $m_{\sigma}$ in MeV. $\sigma$-nucleon $g_{\sigma}$, $\omega$-nucleon $g_{\omega}$, and $\rho$-nucleon $g_{\rho}$ coupling constants used in the calculation. Compressibility $K$ in $\mathrm{MeV}$ and the effective nuclon mass $M^{*} / M$ are given for different $\lambda_{R}$. It is seen that the compressibility decreases and effective mass increases with the increase of $\lambda_{R}$. Note that the calculations have been done with renormalized sigma mass $m_{R}=520$ and $480 \mathrm{MeV}$.

\begin{tabular}{|c|c|c|c|c|c|c|c|c|c|}
\hline$M$ & $m_{\omega}$ & $m_{\rho}$ & $m_{\sigma}$ & $\lambda_{R}$ & $g_{\sigma}$ & $g_{\omega}$ & $g_{\rho}$ & $K$ & $M^{*} / M$ \\
\hline & & & & No-Sea & 9.05 & 11.671 & 5.7704 & 545 & 0.56 \\
\hline & & & & RHA & 7.4975 & 8.9305 & 6.6643 & 450 & 0.72 \\
\hline & & & & 1.8 & 7.1669 & 8.1897 & 7.1303 & 399.4791 & 0.7531 \\
\hline \multirow[t]{3}{*}{939.0} & 783.0 & 770.0 & 520.0 & 3.0 & 6.9276 & 7.6474 & 7.2304 & 364.4575 & 0.7769 \\
\hline & & & & 4.0 & 6.7200 & 7.1671 & 7.3085 & 340.9642 & 0.7965 \\
\hline & & & & 4.5 & 6.6128 & 6.9147 & 7.3460 & 330.6333 & 0.8063 \\
\hline \multirow[t]{4}{*}{939.0} & 783.0 & 770.0 & 480.0 & 1.8 & 6.5169 & 7.9471 & 7.1768 & 382.0059 & 0.7639 \\
\hline & & & & 3.0 & 6.2515 & 7.2837 & 7.2905 & 344.4022 & 0.7919 \\
\hline & & & & 4.0 & 6.0380 & 6.7300 & 7.3720 & 320.1296 & 0.8100 \\
\hline & & & & 4.5 & 5.9332 & 6.4502 & 7.4092 & 309.7436 & 0.8234 \\
\hline
\end{tabular}

teraction. The neutron star gravitational mass limit obtained with this interaction is $1.6 M_{\odot}$. However, boost corrections to the two-nucleon interaction, which give the leading relativistic effect of order $(v / c)^{2}$, as well as three-nucleon interaction increase the mass limit.

In Fig. 3, we plot the angular velocity versus mass of the neutron star. The maximum mass decreases with the increase of $\lambda_{R}$. In Fig. 4, the angular momentum versus mass of the neutron star is plotted. The Walecka model (no-sea) predicts much higher angular momentum for the neutron star. But the angular momentum decreases with the increase of $\lambda_{R}$.

In Figs. 5 and 6 we have plotted radius and angular ve-

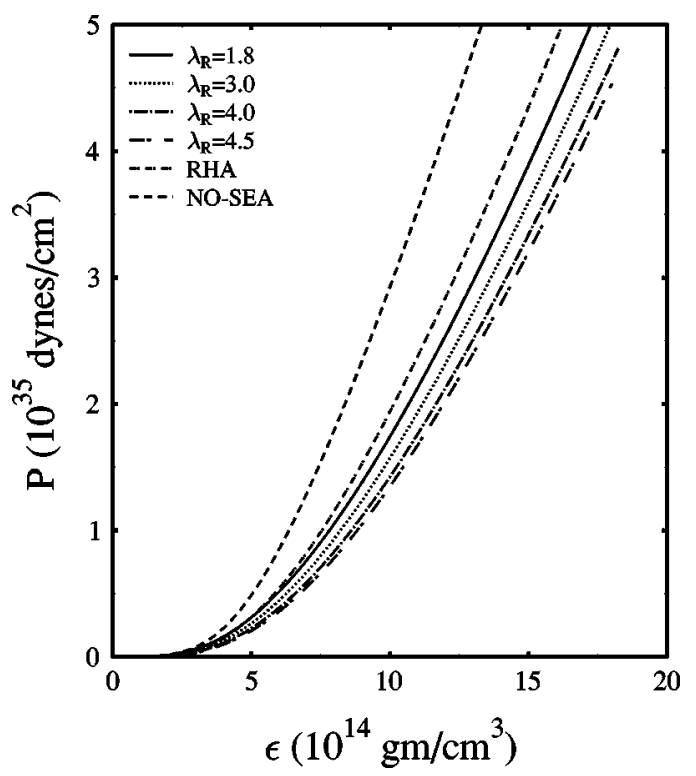

FIG. 1. The equation of state of neutron matter for $m_{R}$ $=520 \mathrm{MeV}$ for different $\lambda_{R}$. The Walecka model (no-sea) approximation gives a stiffer equation of state compared to the other equations of state. locity versus mass for $m_{R}=480 \mathrm{MeV}$. We found that for this value of $m_{R}$, the radius, mass, and angular velocity decrease compared to the result for $m_{R}=520 \mathrm{MeV}$.

\section{CONCLUSIONS}

We have studied the properties of the rotating neutron star using the Walecka model with quantum corrections for baryons and sigma mesons through a realignment of the vacuum. In this study, the vacuum polarization correction is included in nuclear matter in a nonperturbative approach. In this approach, the contribution from the ground-state structure with baryon-antibaryon condensates yields the same results as obtained through a relativistic Hartree approximation of summing tadpole diagrams for the baryon propagator. This results in a softer equation of state. This vacuum is then generalized by including the quantum effects from $\sigma$-meson

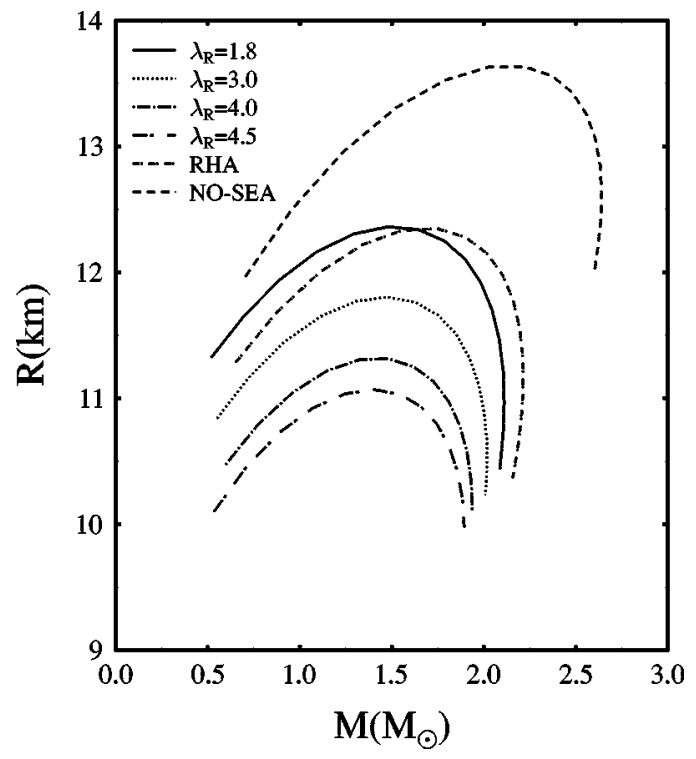

FIG. 2. The radius versus mass of the neutron star for $m_{R}$ $=520 \mathrm{MeV}$ for different $\lambda_{R}$. 


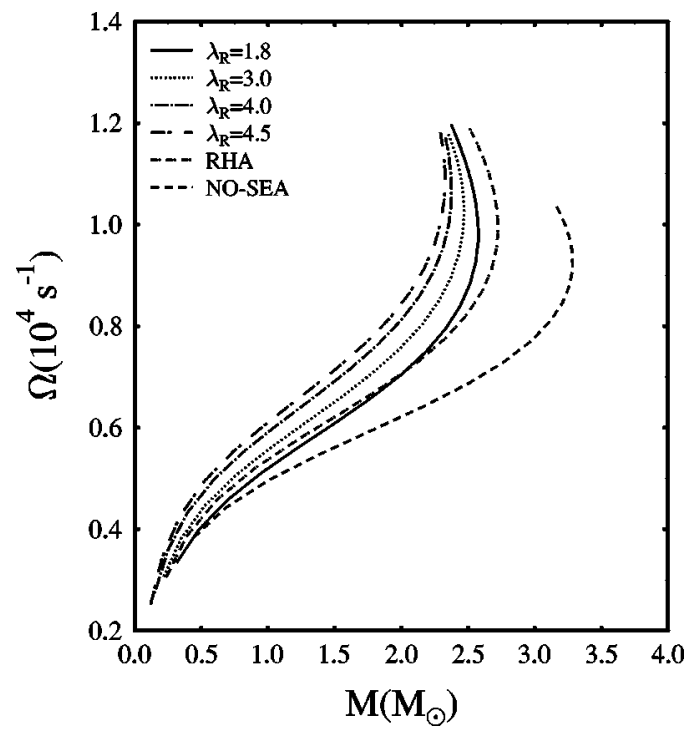

FIG. 3. The angular velocity versus mass of the neutron star for $m_{R}=520 \mathrm{MeV}$ for different $\lambda_{R}$.

fields through scalar-meson condensates, which amounts to summing over a class of multiloop diagrams. This leads to a further softening of the equation of states. The value of the compressibility and the effective mass are within the range of 300-400 MeV and 0.75-0.8, respectively. It is interesting to note that the present variational ansatz with squeezed vacuum structure leads to daisy-superdaisy resummed selfconsistent two-loop effective potentials as obtained in Ref [17]. The reason for such a result lies in the fact that the $\sigma^{4}$ interaction leads to a functional for the vacuum energy which is effectively quadratic and we could solve for the ansatz functions explicitly. It is known that most of the successful parameter sets which explain the ground-state properties of nuclear matter and finite nuclei quite well are with a negative quartic coupling. But the energy spectrum in such a case is

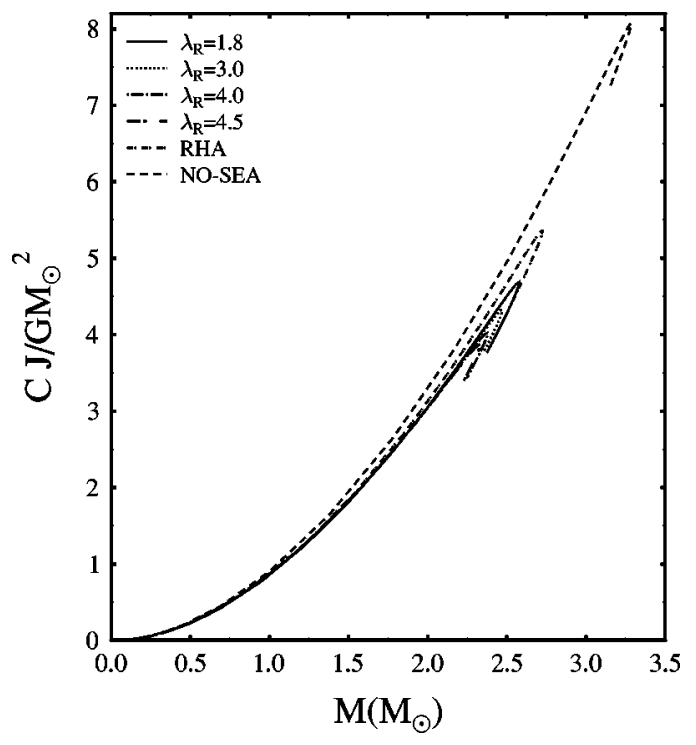

FIG. 4. The angular momentum versus mass of the neutron star for $m_{R}=520 \mathrm{MeV}$ for different $\lambda_{R}$.

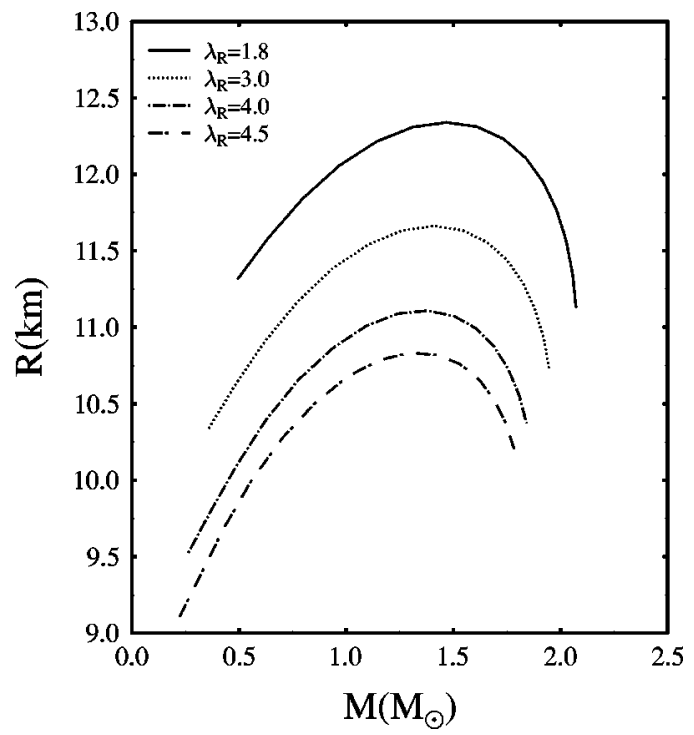

FIG. 5. The radius versus mass of the neutron star for $m_{R}$ $=480 \mathrm{MeV}$ for different $\lambda_{R}$. Note that the radius and mass decrease as obtained for $m_{R}=520 \mathrm{MeV}$.

unbounded from below for large $\sigma$, thus making it impossible to include vacuum-polarization effects. We have included quantum effects with a quartic self-interaction through $\sigma$ condensates, taking the coupling to be positive. We then extended the model by including the $\rho$ mesons and applied it to study the properties of neutron stars. However, the renormalization of $\omega$ and $\rho$ mesons has been neglected.

We reemphasized the role of the equation of state in neutron star structure. In our calculation for neutron matter at zero temperature, we found that the Walecka model (no sea) gives a stiffer equation of state and the compressibility is quite large. However, the equation of state becomes much softer with the increase of renormalized coupling $\lambda_{R}$ for a given renormalized mass $m_{R}$. We calculated neutron star

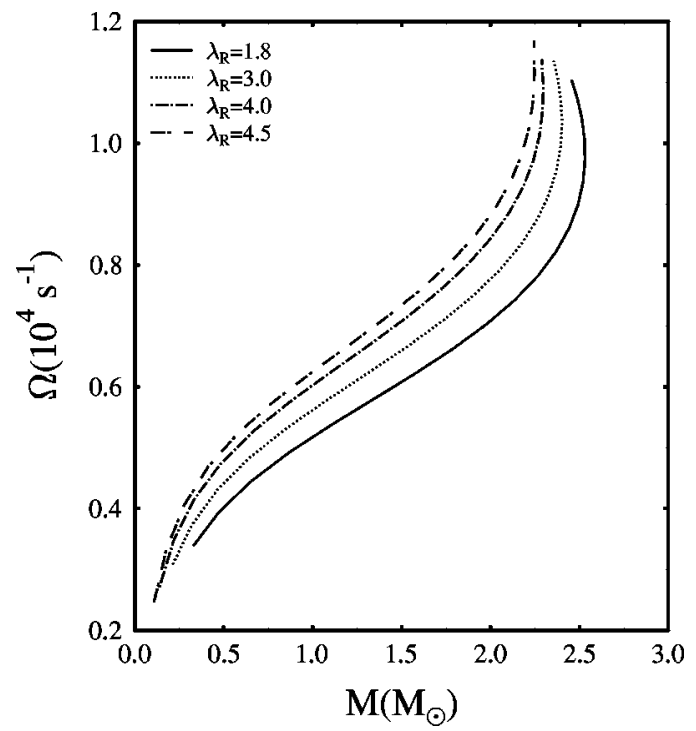

FIG. 6. The angular velocity versus mass of the neutron star for $m_{R}=480 \mathrm{MeV}$ for different $\lambda_{R}$. 
properties like mass, radius, and angular velocity which may become tests of the theory as more data on neutron stars become available. We found that for $m_{R}=480 \mathrm{MeV}$, the radius, mass, and angular velocity decrease compared to the result for $m_{R}=520 \mathrm{MeV}$.

Note that the approximation here lies in the specific ansatz for the ground-state structure. However, a systematic inclusion of more general condensates than the pairing one as used here might be an improvement over the present one.

\section{ACKNOWLEDGMENTS}

One of the authors (P.K.P.) would like to acknowledge FAPESP (Processo-99/08544-0) for financial support and the IFT, Sao Paulo, for kind hospitality. P.K.P. would also like to thank G. Krein for helpful conversations about this work. R.S. acknowledges the Department of Science and Technology, Government of India, for financial help.
[1] B.D. Serot and J.D. Walecka, Adv. Nucl. Phys. 16, 1 (1986); Int. J. Mod. Phys. E 6, 515 (1997); J.D. Walecka, Ann. Phys. (N.Y.) 83, 491 (1974).

[2] T.D. Cohen, Phys. Rev. C 45, 833 (1992).

[3] N.K. Glendenning, Nucl. Phys. A493, 521 (1989).

[4] P.J. Ellis, S.B. Parendo, and M. Prakash, Phys. Lett. B 361, 5 (1995).

[5] R.J. Furnstahl, R.J. Perry, and B.D. Serot, Phys. Rev. C 40, 321 (1989).

[6] A. Mishra, P.K. Panda, S. Schramm, J. Reinhardt, and W. Greiner, Phys. Rev. C 56, 1380 (1997).

[7] H. Mishra, S.P. Misra, and A. Mishra, Int. J. Mod. Phys. A 3, 2331 (1988).

[8] A. Mishra, H. Mishra, and S.P. Misra, Z. Phys. C 59, 159 (1993).

[9] S.P. Misra, Phys. Rev. D 18, 1661 (1978).

[10] H. Umezawa, H. Matsumoto, and M. Tachiki, Thermofield Dynamics and Condensed States (North-Holland, Amsterdam, 1982); Y. Takahashi and H. Umezawa, Int. J. Mod. Phys. B 10, 1755 (1996); P.A. Henning, Phys. Rep. 253, 2355 (1995).

[11] S.A. Chin and J.D. Walecka, Phys. Lett. 52B, 24 (1974); S.A. Chin, Ann. Phys. (N.Y.) 108, 301 (1977); R.J. Perry, Phys. Lett. B 199, 489 (1987).
[12] J. Boguta and A.R. Bodmer, Nucl. Phys. A292, 413 (1977).

[13] M. Rufa, P.G. Reinhard, J. Maruhn, and W. Greiner, Phys. Rev. C 38, 390 (1988); Y.K. Gambhir, P. Ring, and A. Thimet, Ann. Phys. (N.Y.) 198, 132 (1990); Y. Sugahara and H. Toki, Nucl. Phys. A574, 557 (1994).

[14] R.J. Furnstahl, C.E. Price, and G.E. Walker, Phys. Rev. C 36, 2590 (1987).

[15] A. Mishra and H. Mishra, J. Phys. G 23, 143 (1997).

[16] S. Coleman, R. Jackiw, and H.D. Politzer, Phys. Rev. D 10, 2491 (1974).

[17] S.Y. Pi and M. Samiullah, Phys. Rev. D 36, 3128 (1987); G. A. Camelia and S.Y. Pi, ibid. 47, 2356 (1993).

[18] H. Komatsu, Y. Eriguchi, and I. Hachisu, Mon. Not. R. Astron. Soc. 237, 355 (1989).

[19] G.B. Cook, S.L. Shapiro, and S.A. Teukolsky, Astrophys. J. 422, 227 (1994), and references therein.

[20] N. Stergioulas and J.L. Friedman, Astrophys. J. 444, 306 (1995).

[21] J.M. Weisenberg and J.H. Taylor, Phys. Rev. Lett. 52, 1348 (1984).

[22] A. Akmal, V.R. Pandharipande, and D.G. Ravenhall, Phys. Rev. C 58, 1804 (1998). 